PROCEEDINGS OF THE

AMERICAN MATHEMATICAL SOCIETY

Volume 136, Number 7, July 2008, Pages 2407-2415

S 0002-9939(08)09286-1

Article electronically published on February 20, 2008

\title{
SINGULARITIES OF GENERIC PROJECTION HYPERSURFACES
}

\author{
DAVIS C. DOHERTY
}

(Communicated by Ted Chinburg)

\begin{abstract}
Linearly projecting smooth projective varieties provide a method of obtaining hypersurfaces birational to the original varieties. We show that in low dimension, the resulting hypersurfaces only have Du Bois singularities. Moreover, we conclude that these $\mathrm{Du}$ Bois singularities are in fact semi log canonical. However, we demonstrate the existence of counterexamples in high dimension - the generic linear projection of certain varieties of dimension 30 or higher is neither semi log canonical nor Du Bois.
\end{abstract}

\section{INTRODUCTION}

The study of smooth projective curves over $\mathbb{C}$ is greatly simplified by the classical result that every such curve is birational to a plane curve having only nodal singularities, with the birational morphism given by a generic linear projection. Applying an analogous technique to higher-dimensional varieties seems a natural extension - can we draw any conclusions in this more general setting? Joel Roberts (Rob71], Rob75]) provides a useful starting point for understanding the singularities introduced by linearly projecting varieties. Applying the more recent machinery of birational geometry to his work offers the possibility of finding a useful answer to this question.

Our approach is as follows: start with a smooth projective variety, obtain a birational projective hypersurface by taking a (generic) linear projection, and classify the singularities of the resulting hypersurface. The principal challenge is in determining the type of singularities introduced by the projection - in order for this approach to be useful, we would like to constrain these to a reasonably nice class. To that end, we initially propose that the appropriate class of singularities is semi log canonical (slc) - these are the singularities appearing on the boundaries of moduli spaces for most higher-dimensional moduli problems.

Unfortunately, determining semi log canonicity is highly nontrivial even for hypersurfaces. To that end, the somewhat obscure class of Du Bois singularities serves as a useful tool, as we establish in the following generalization of a theorem due to Sándor Kovács ( Kov99, Thm 3.6]).

Theorem 4.2. Let $X$ be an $S_{2}$ scheme which is semismooth in codimension one, and assume that $K_{X}$ is Cartier and $X$ has Du Bois singularities. Then $X$ is semi log canonical.

Received by the editors June 11, 2007, and, in revised form, June 20, 2007.

2000 Mathematics Subject Classification. Primary 14J17; Secondary 14E15, 14B05.

(C)2008 American Mathematical Society Reverts to public domain 28 years from publication 2407 
Applying a variety of techniques to the results of Rob75, combined with the above, leads to the following extension of the classical result on curves.

Main Theorem. Let $Y \subset \mathbb{P}^{N}$ be a smooth projective variety of dimension n, $n \leq 5$, embedded via the $d$-uple embedding with $d \geq 3 n$. Let $X \subset \mathbb{P}^{n+1}$ be the image of $Y$ under a generic projection $\pi: Y \rightarrow \mathbb{P}^{n+1}$. Then $X$ has semi log canonical singularities.

Though the dimensional restrictions in the Main Theorem seem arbitrary, the statement cannot be generalized under these assumptions: counterexamples exist in high dimensions.

Corollary 4.7. Let $X \subset \mathbb{P}^{31}$ be a generic projection hypersurface obtained via $\pi: Y \rightarrow \mathbb{P}^{31}$, where $\Omega_{Y}^{1}$ is nef. Then $X$ is not semi log canonical.

I would like to express my gratitude to Sándor Kovács, who advised me throughout this work, Karl Schwede, whose help with Du Bois singularities was instrumental, and Rob Lazarsfeld, who pointed out that the counterexamples of Corollary 4.7 should exist.

\section{Definitions And Conventions}

Throughout this work we assume all schemes to be separated of finite type over $\mathbb{C}$. A variety is a reduced and separated scheme of finite type over $\mathbb{C}$. A scheme is Gorenstein if all its local rings are Gorenstein rings. Similarly, scheme is $S_{2}$ if all its local rings satisfy Serre's $S_{2}$ condition. A scheme (or more generally, an algebraic space) $X$ of dimension $n$ is semismooth if every closed point is either smooth, a double normal crossing point - analytically isomorphic to $\mathbb{C}\left[x_{0}, \ldots, x_{n}\right] /\left(x_{0} x_{1}\right)$ or a pinch point - analytically isomorphic to $\mathbb{C}\left[x_{0}, \ldots, x_{n}\right] /\left(x_{0} x_{1}^{2}-x_{2}^{2}\right)$ (note that a semismooth scheme is Gorenstein). Its double locus $D_{X}$ is the codimension one subscheme of non-smooth points. A proper birational map $f: Y \rightarrow X$ is called a semiresolution if $Y$ is semismooth, no component of its double locus $D_{Y}$ is in the exceptional locus of $f$, and there is a codimension two subset $S \subset X$ such that the restriction map $f^{-1}(X \backslash S) \rightarrow X \backslash S$ is an isomorphism. $f$ is a good semiresolution if, in addition, $E \cup D_{Y}$ is a simple normal crossing divisor (where $E$ is the exceptional divisor of $f$ ). By [Kol90, Prop 4.2] semiresolutions always exist, as long as we're willing to work in the category of algebraic spaces.

Now suppose $X$ is a reduced $S_{2}$ scheme which is semismooth in codimension one. We say that $X$ has semi log canonical singularities if $K_{X}$ is $\mathbb{Q}$-Cartier and there is a good semiresolution $f: Y \rightarrow X$ such that

$$
K_{Y} \equiv f^{*} K_{X}+\sum_{i} a_{i} E_{i}
$$

with all $a_{i} \geq-1$, where $E_{i}$ are the exceptional divisors (see [Kol92, §12] for details).

2.1. Du Bois singularities. Denote by $D_{\text {filt }}(X)$ the bounded, filtered derived category of sheaves on a scheme $X$ with coherent cohomology; this is the only derived category we shall consider, so this abbreviated notation will be unambiguous. We denote quasi-isomorphisms by $\simeq_{\text {qis }}$.

Philippe Du Bois (DB81] demonstrated the existence of an object $\underline{\Omega}_{X} \in D_{\text {filt }}(X)$ (the $D u$ Bois complex) with the following properties: 
(i) If $\Omega_{X}$ denotes the usual De Rham complex with the "filtration bête", then there is a natural morphism $\Omega_{X} \rightarrow \underline{\Omega}_{X}$ in the filtered derived category (i.e., respecting the filtrations); if $X$ is smooth, this map is a quasi-isomorphism.

(ii) The Du Bois complex is local in the étale topology - if $U$ is an étale open set, then $\left.\underline{\Omega}_{X}\right|_{U} \simeq_{\text {qis }} \underline{\Omega}_{U}$.

(iii) If $f: Y \rightarrow X$ is a proper morphism, then there is a natural morphism $f^{*}$ : $\underline{\Omega}_{X} \rightarrow R f_{*} \underline{\Omega}_{Y}$ in the filtered derived category.

(iv) Let $f: Y \rightarrow X$ be proper, and assume that $f$ is an isomorphism outside a closed subscheme $\Sigma \subset X$, with reduced preimage $f^{-1}(\Sigma)=E$. Then there exists an exact triangle

$$
\underline{\Omega}_{X} \rightarrow \underline{\Omega}_{\Sigma} \oplus R f_{*} \underline{\Omega}_{Y} \rightarrow R f_{*} \underline{\Omega}_{E} \stackrel{+1}{\longrightarrow} .
$$

Set $\underline{\Omega}_{X}^{0}=\mathrm{Gr}_{\text {filt }}^{0} \underline{\Omega}_{X}$. There is a natural morphism $\mathscr{O}_{X} \rightarrow \underline{\Omega}_{X}^{0}$; we say that $X$ has $\mathrm{Du}$ Bois singularities if this is a quasi-isomorphism.

\section{Identifying Du Bois singularities}

Determining when a scheme has Du Bois singularities is a difficult problem. In this section, we review some known methods for identifying Du Bois singularities, and provide some new ones. Steenbrink's following result is one of the most useful.

Theorem 3.1 (Ste81, Thm 3]). Let $X$ be a variety, with $\pi: \widetilde{X} \rightarrow X$ its normalization and $\mathscr{C}=\operatorname{Ann}_{\mathscr{O}_{X}}\left(\pi_{*} \mathscr{O}_{\widetilde{X}} / \mathscr{O}_{X}\right)$ the conductor ideal sheaf of the map $\pi$. Define $\Sigma \subseteq X$ to be the subvariety defined by $\mathscr{C}$, and let $E=\pi^{-1}(\Sigma)$. Suppose $\widetilde{X}, E$ and $\Sigma$ all have Du Bois singularities. Then $X$ has Du Bois singularities.

Example 3.2. The pinch point is a Du Bois singularity. The normalization of its coordinate ring is $\mathbb{C}\left[y_{1}, y_{2}, x_{4}, \ldots, x_{n}\right]$, which defines a smooth (and therefore Du Bois) scheme. The normalization map is given by

$$
\begin{aligned}
x_{1} & \mapsto y_{1} y_{2}, \\
x_{2} & \mapsto y_{2}, \\
x_{3} & \mapsto y_{1}^{2}, \\
x_{i} & \mapsto x_{i}, i \geq 4 .
\end{aligned}
$$

The conductor is the ideal $\left(x_{1}, x_{2}\right)$, which defines a smooth subscheme. To obtain the preimage of this subscheme, we take the image of the conductor in the normalization, which is the ideal $\left(y_{2}\right)$; thus we can apply the theorem.

Example 3.3. A double normal crossing singularity is also Du Bois. The normalization of its coordinate ring is $\mathbb{C}\left[x_{1}, \ldots, x_{n}\right] /\left(x_{1}\right) \oplus \mathbb{C}\left[x_{1}, \ldots, x_{n}\right] /\left(x_{2}\right)$, which defines a smooth variety. The conductor is given by the ideal $\left(x_{1}, x_{2}\right)$, which defines a smooth subscheme; the preimage of this subscheme is the direct sum of two copies of $\mathbb{C}\left[x_{1}, \ldots, x_{n}\right] /\left(x_{1}, x_{2}\right)$, which is again smooth.

Pinch points and double normal crossing points are the only singularities of semismooth schemes, so the above examples give the following.

Proposition 3.4. If $X$ is semismooth, then $X$ is Du Bois.

Karl Schwede recently proved the following interesting connection between Finjective and Du Bois singularities (see Fed83] and many others for details on F-injective and F-pure singularities). 
Theorem 3.5 ([Sch06, Thm. 6.4.3]). Suppose that $X$ is a variety over $\mathbb{C}$ of dense F-injective type. Then $X$ has Du Bois singularities.

Combined with [Fed83, Prop 2.1] and [Fed83, Lemma 3.3], this gives the corollary we shall use:

Corollary 3.6. Suppose $X=V(f)$ is a variety defined (locally) by a single equation $f \in \mathbb{C}\left[x_{1}, \ldots, x_{n}\right]$. Assume $P=(0, \ldots, 0) \in X$ is a singular point. If $f^{p-1} \notin$ $\left(x_{1}^{p}, \ldots, x_{n}^{p}\right)$ for all but finitely many primes $p$, then $P \in X$ is a Du Bois singularity.

3.1. Products of Du Bois schemes. Does a product of schemes with Du Bois singularities also have Du Bois singularities? This is a natural question to ask, but seems to be missing from the scant literature on this class of singularities. Such a "product theorem" is the final piece required before proceeding to the proof of the Main Theorem.

One more type of singularity plays a role in the product theorem.

Definition 3.7. If $X$ is a variety, we say that $X$ has generalized simple normal crossings if for each singular point $x \in X$ we have an analytic isomorphism

$$
\widehat{\mathscr{O}}_{X, x} \cong \mathbb{C}\left[\left[x_{1}, \ldots, x_{n}\right]\right] /\left(I_{1} \cap \cdots \cap I_{k}\right),
$$

where each $I_{k}$ is generated by coordinate functions, i.e., $I_{k}=\left(x_{k_{1}}, \ldots, x_{k_{j}}\right)$. Note that if $X$ and $Y$ have generalized simple normal crossings, then $X \times Y$ also has generalized simple normal crossings.

Lemma 3.8. Suppose that $X$ has generalized simple normal crossings. Then $X$ has Du Bois singularities.

Proof. Note the following "gluing" fact of Du Bois singularities: if $Y$ is a variety over $\mathbb{C}$ with components $Y_{1}$ and $Y_{2}$ such that $Y_{1}, Y_{2}$ and $Y_{1} \cap Y_{2}$ have Du Bois singularities, then $Y$ has Du Bois singularities (Sch06, Thm. 5.2.1]).

We proceed by induction on the maximum dimension of a component and the number of components. If $X$ is the union of two components of any dimension, it has $\mathrm{Du}$ Bois singularities by the gluing property (here the intersection is actually smooth). Now assume that the conclusion holds when all components are less than dimension $d$, and that it holds for $n$ components of dimension $d$. Suppose that we have $n+1$ components of dimension at most $d$. Then $X$ can be expressed as the union of the components $X_{1}$ and $X_{2}$, where $X_{1}$ is the union of the first $n$ components and $X_{2}$ is the last component. By the induction hypothesis $X_{1}$ is Du Bois and $X_{2}$ is smooth. $X_{1} \cap X_{2}$ has generalized simple normal crossing singularities and components of dimension $d-1$ or less, so by the induction hypothesis it also has Du Bois singularities. By the gluing property, we conclude that $X$ has Du Bois singularities.

Theorem 3.9. Suppose $X_{1}$ and $X_{2}$ are varieties (over $\mathbb{C}$ ) which have Du Bois singularities. Then $X_{1} \times X_{2}$ also has Du Bois singularities.

Proof. The property of having Du Bois singularities is local (in the étale topology), so by restricting to affine open sets we may assume that $X_{1}$ and $X_{2}$ are affine. Embed each $X_{i}$ into a smooth variety $Y_{i}$, and let $f_{i}: \widetilde{Y}_{i} \rightarrow Y_{i}$ be a strong $\log$ resolution of $X_{i}$. Denote by $E_{i}$ the (reduced) pre-image of $X_{i}$. By [Sch06, Thm. 5.3.4], we have $\underline{\Omega}_{X_{i}}^{0} \simeq_{\text {qis }} R f_{i *} \mathscr{O}_{E_{i}}$. Since $X_{i}$ was assumed to have Du Bois singularities, we have $\mathscr{O}_{X_{i}} \simeq_{\text {qis }} R f_{i *} \mathscr{O}_{E_{i}}$. 
Note that $\widetilde{Y}_{1} \times \widetilde{Y}_{2}$ is smooth (hence has rational singularities) and $g$ is an isomorphism outside of $X_{1} \times X_{2}$. By assumption, $E_{1}$ and $E_{2}$ have generalized simple normal crossings. Hence $E_{1} \times E_{2}$ has generalized simple normal crossings, and so has Du Bois singularities by Lemma 3.8. In particular, this means that $R g_{*} \mathscr{O}_{E_{1} \times E_{2}} \simeq_{\text {qis }} \underline{\Omega}_{X_{1} \times X_{2}}^{0}$ by [Sch06, Thm. 5.3.4].

Now consider the following diagram:

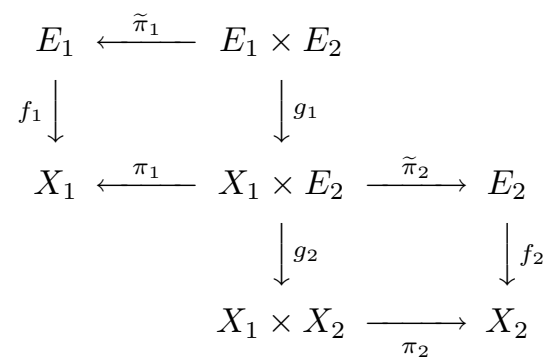

(Note that $g_{1}$ and $g_{2}$ are the obvious maps such that $g_{2} \circ g_{1}=\left.g\right|_{E_{1} \times E_{2}}$, and $\widetilde{\pi}_{i}$ and $\pi_{i}$ are the usual projection maps.) Applying [Har77, Thm. III.9.3] to the upper left square in the diagram, we find that $\pi_{1}^{*} R f_{1 *} \mathscr{O}_{E_{1}} \cong R g_{1 *}\left(\widetilde{\pi}_{1}^{*} \mathscr{O}_{E_{1}}\right)$. Since $\widetilde{\pi}_{1}$ is projection onto a factor, we have $\widetilde{\pi}_{1}^{*} \mathscr{O}_{E_{1}} \cong \mathscr{O}_{E_{1} \times E_{2}}$; by assumption, $R f_{1 *} \mathscr{O}_{E_{1}} \simeq_{\text {qis }} \mathscr{O}_{X_{1}}$, so we obtain $R g_{1 *}\left(\mathscr{O}_{E_{1} \times E_{2}}\right) \simeq_{\text {qis }} \pi_{1}^{*} \mathscr{O}_{X_{1}} \cong \mathscr{O}_{X_{1} \times E_{2}}$ (the last isomorphism is again due to the fact that $\pi_{1}$ is a projection map). Applying this same argument again to the lower right square gives $R g_{2 *} \mathscr{O}_{X_{1} \times E_{2}} \simeq_{\text {qis }} \mathscr{O}_{X_{1} \times X_{2}}$. Thus we see that $R g_{*} \mathscr{O}_{E_{1} \times E_{2}} \simeq_{\text {qis }} \mathscr{O}_{X_{1} \times X_{2}}$; combined with the above, we see that $\mathscr{O}_{X_{1} \times X_{2}} \simeq_{\text {qis }} \underline{\Omega}_{X_{1} \times X_{2}}^{0}$, so that $X_{1} \times X_{2}$ is Du Bois.

\section{Singularities of GeneriC PROJECTIONS}

4.1. Proof of the Main Theorem. Recall that the set of linear projections $\mathbb{P}^{m} \rightarrow$ $\mathbb{P}^{r}$ is in bijection with the $(m-r)$-dimensional linear subspaces of $\mathbb{P}^{m}$, and can thus be identified with the closed points of the Grassmannian variety $G(m, m-r-1)$. We say that a generic projection has property $P$ if the collection of points with property $P$ forms an open dense subset of the Grassmannian. With this in mind, we proceed to the proof of the Main Theorem.

Main Theorem. Let $Y \subset \mathbb{P}^{N}$ be a smooth projective variety of dimension $n$, $n \leq 5$, embedded via the d-uple embedding with $d \geq 3 n$. Let $X \subset \mathbb{P}^{n+1}$ be the image of $Y$ under a generic projection $\pi: Y \rightarrow \mathbb{P}^{n+1}$. Then $X$ has Du Bois singularities:

Proof. [Rob75, §13.2] provides a list of the possible local analytic isomorphism classes of the singularities that arise from such a generic projection. Since the Du Bois complex is local in the étale toplogy, it will suffice to show that in each case these define $\mathrm{Du}$ Bois singularities.

Case (0) Let $R=\mathbb{C}\left[\left[x_{1}, \ldots, x_{n+1}\right]\right] /\left(x_{1} \cdots x_{d}\right)$. In this case, we have $f_{1}=x_{1} \cdots x_{d}$. Reducing to characteristic $p$, we see that $f_{1}^{p-1}=x_{1}^{p-1} \cdots x_{n}^{p-1}$ and $\mathfrak{m}^{[p]}=$ $\left(x_{1}^{p}, \ldots, x_{n+1}^{p}\right)$. We clearly have $f_{1}^{p-1} \notin \mathfrak{m}^{[p]}$ for all primes $p$, so $R$ has Du Bois singularities by Corollary 3.6 .

Case (1a) The pinch point was shown to be Du Bois in Example 3.2. 
Case (1b) Let $R=\mathbb{C}\left[\left[x_{1}, \ldots, x_{n+1}\right]\right] /\left(x_{n}^{3}+\Phi_{4}+\Phi_{5}\right)$, with

$$
\begin{aligned}
& \Phi_{4}=x_{1}^{2} x_{3} x_{n}-x_{1}^{3} x_{n+1}+2 x_{2} x_{3} x_{n}^{2}-3 x_{1} x_{2} x_{n} x_{n+1}, \\
& \Phi_{5}=x_{2}^{2} x_{3}^{2} x_{n}-x_{1} x_{2}^{2} x_{3} x_{n+1}-x_{2}^{3} x_{n+1}^{2} ;
\end{aligned}
$$

denote the full polynomial generating the ideal by $f_{3}$. Examination of the monomials occurring in $f_{3}$ shows that there is a term of the form $\left(-3 x_{1} x_{2} x_{n} x_{n+1}\right)^{p-1}$ in $f_{3}^{p-1}$. No other product of monomials in $f_{3}$ can generate a monomial of the form $\left(x_{1} x_{2} x_{n} x_{n+1}\right)^{k}$, so the coefficient of $\left(x_{1} x_{2} x_{n} x_{n+1}\right)^{p-1}$ is non-zero for $p \neq 3$. Since this monomial is not in $\mathfrak{m}^{[p]}$, it follows that $f_{3} \notin \mathfrak{m}^{[p]}$. Again, Corollary 3.6 implies this point id Du Bois.

Case (2a) The ring $R=\mathbb{C}\left[\left[x_{1}, \ldots, x_{n+1}\right]\right] /\left(x_{1}\left(x_{n}^{2}-x_{2}^{2} x_{n+1}\right)\right)$ is the coordinate ring of a product $X_{1} \times X_{2}$ (where we take $X_{1}$ to be a pinch point and $X_{2}$ to be a hyperplane), hence defines a Du Bois singularity by Theorem 3.9.

Case (2b) $R=\mathbb{C}\left[\left[x_{1}, \ldots, x_{n+1}\right]\right] /\left(x_{1}\left(x_{n}^{3}+\Psi_{4}+\Psi_{5}\right)\right)$, where

$$
\Psi_{i}=\Phi_{i}\left(x_{2}, x_{3}, x_{4}, x_{n}, x_{n+1}\right),
$$

is also Du Bois by Theorem 3.9 and Case (1b).

Case (2c) $R=\mathbb{C}\left[\left[x_{1}, \ldots, x_{n+1}\right]\right] /\left(\left(x_{n}^{2}-x_{1}^{2} x_{n+1}\right)\left(x_{n-2}^{2}-x_{2}^{2} x_{n-1}\right)\right)$ defines a Du Bois singularity by the product theorem and Case (1a).

Case (3) $R=\mathbb{C}\left[\left[x_{1}, \ldots, x_{n+1}\right]\right] /\left(x_{1} x_{2}\left(x_{n}^{2}-x_{3}^{2} x_{n+1}\right)\right)$ is Du Bois by the product theorem and Case (2a).

Case (4) Finally, $R=\mathbb{C}\left[\left[x_{1}, \ldots, x_{n+1}\right]\right] /\left(x_{1} x_{2} x_{3}\left(x_{n}^{2}-x_{4}^{2} x_{n+1}\right)\right)$ is Du Bois by the product theorem and Case (3).

4.2. Du Bois versus semi log canonical. With a few additional results, we can use the Main Theorem to address semi log canonicity of generic projection hypersurfaces. Toward that end, we demonstrate that Du Bois singularities are in fact semi log canonical under certain additional assumptions.

Lemma 4.1. Let $f: Y \rightarrow X$ be a good semiresolution. Assume that $\pi$ is an isomorphism outside a closed subscheme $\Sigma \subset X$, with preimage $f^{-1}(\Sigma)=E$. Then there exists an exact triangle

$$
\underline{\Omega}_{X}^{0} \rightarrow \underline{\Omega}_{\Sigma}^{0} \oplus R f_{*} \mathscr{O}_{Y} \rightarrow R f_{*} \mathscr{O}_{E} \stackrel{+1}{\longrightarrow} .
$$

Proof. Since $Y$ is semismooth, $\underline{\Omega}_{Y}^{0} \simeq_{\text {qis }} \mathscr{O}_{Y}$. The assumption that $f$ is a good semiresolution means that $E$ has only normal crossing singularities, so that $\underline{\Omega}_{E}^{0} \simeq_{\text {qis }}$ $\mathscr{O}_{E}$. The result then follows from [DB81, Prop. 4.11].

Theorem 4.2. Let $X$ be an $S_{2}$ scheme which is semismooth in codimension one, and assume that $K_{X}$ is Cartier and $X$ has Du Bois singularities. Then $X$ is semi $\log$ canonical.

Proof. Let $f: Y \rightarrow X$ be a good semiresolution of $X$, with $W \subseteq X$ the set outside which $f$ is an isomorphism, and $E=f^{-1}(S)$. There exists a natural morphism $\phi: R f_{*} \mathscr{O}_{Y}(-E) \rightarrow R f_{*} \mathscr{O}_{Y}$ arising from the short exact sequence

$$
0 \rightarrow \mathscr{O}_{Y}(-E) \rightarrow \mathscr{O}_{Y} \rightarrow \mathscr{O}_{E} \rightarrow 0
$$

We note that $R f_{*} \mathscr{O}_{Y}(-E) \rightarrow R f_{*} \mathscr{O}_{E}$ is the zero map, from which it follows (via the exact triangle in Lemma 4.1) that $\phi$ factors through $\underline{\Omega}_{X}^{0}$. By assumption, 
$\underline{\Omega}_{X}^{0} \simeq_{\text {qis }} \mathscr{O}_{X}$, so we obtain a morphism $R f_{*} \mathscr{O}_{Y}(-E) \rightarrow \mathscr{O}_{X}$ which is a quasiisomorphism on $X \backslash S$. Applying $R \mathscr{H} o m_{X}\left(-, \omega_{X}\right)$, we obtain a morphism $\omega_{X} \rightarrow$ $R \mathscr{H} 0 m_{X}\left(R f_{*} \mathscr{O}_{Y}(-E), \omega_{X}^{\prime}\right)$. But then we have

$$
\begin{aligned}
R \mathscr{H} \operatorname{om}_{X}\left(R f_{*} \mathscr{O}_{Y}(-E), \omega_{X}\right) & \cong R f_{*} R \mathscr{H} \operatorname{om}_{Y}\left(\mathscr{O}_{Y}(-E), \omega_{Y}\right) \\
& \cong R f_{*}\left(R \mathscr{H} \operatorname{om}_{Y}\left(\mathscr{O}_{Y}(-E), \mathscr{O}_{Y}\right) \otimes \omega_{Y}\right),
\end{aligned}
$$

where the first isomorphism follows from Grothendieck duality, and the second follows from the fact that $Y$ is Gorenstein. Since $E$ is a Cohen-Macaulay divisor (it has only simple normal crossings), this last term is isomorphic to $R f_{*} \omega_{Y}(E)[n]$, so in fact we have a morphism $\omega_{X} \rightarrow R f_{*} \omega_{Y}(E)[n]$. Taking the $-n$th cohomology gives a morphism $\omega_{X} \rightarrow f_{*} \omega_{Y}(E)$ which is an isomorphism on $X \backslash S$. Adjointness produces a non-zero morphism $f^{*} \omega_{X} \rightarrow \omega_{Y}(E)$. Since $f^{*} \omega_{X}$ is a line bundle this implies $f^{*} \omega_{X} \subseteq \omega_{Y}(E)$, whence $X$ is semi log canonical.

Corollary 4.3. Let $X$ be a seminormal Gorenstein scheme with Du Bois singularities. Then $X$ is semi log canonical.

Proof. The Gorenstein assumption implies that $K_{X}$ is Cartier, and also that $X$ is G1, i.e., $X$ is $S_{2}$ and Gorenstein in codimension 1. By Theorem GT80, Thm 9.10], a seminormal G1 scheme is semismooth in codimension one. The result then follows immediately from the theorem.

Corollary 4.4. Let $X$ be as in the Main Theorem. Then $X$ has semi log canonical singularities.

Proof. Since $X$ is a hypersurface, it is a complete intersection; in particular, $X$ is Gorenstein. By GT80, Thm 3.7], $X$ is seminormal, so Corollary 4.3 implies that $X$ is semi log canonical.

4.3. Counterexamples in higher dimensions. Ideally we would like a more general result that generic projection hypersurfaces are always Du Bois (and thus semi log canonical). The following results illustrate that counterexamples exist, though all known examples have high dimension.

Theorem 4.5. Suppose $X \subset \mathbb{P}^{n+1}$ is a hypersurface, and that there exists some point $x \in X$ having multiplicity $\mu>n+1$. Then $X$ is not semi log canonical.

Proof. Let $f: Z \rightarrow \mathbb{P}^{n+1}$ be the blow-up at $x$, and let $g: X^{\prime} \rightarrow X$ be the restriction of $f$ to the strict transform of $X$. Since $f$ is a blow-up at a point, we have (by [Har77, Ex. II.8.5])

$$
K_{Z} \equiv f^{*} K_{\mathbb{P}^{n+1}}+n E .
$$

Similarly, by the definition of $X^{\prime}$ and our choice of $x$ we have

$$
X^{\prime}=g^{*} X-\left.\mu E\right|_{X^{\prime}}
$$

where we are abusing notation and identifying $X$ and $X^{\prime}$ with the corresponding divisors. Applying the adjunction formula, we obtain

$$
\begin{aligned}
K_{X^{\prime}} & \left.\equiv\left(K_{Z}+X^{\prime}\right)\right|_{X^{\prime}} \\
& \left.\equiv\left(f^{*} K_{\mathbb{P}}+X^{\prime}+n E\right)\right|_{X^{\prime}} \\
& \left.\equiv\left(f^{*} K_{\mathbb{P}}+g^{*} X-\left.\mu E\right|_{X^{\prime}}+n E\right)\right|_{X^{\prime}} \\
& \equiv f^{*}\left(K_{\mathbb{P}}+X\right)+\left.(n-\mu) E\right|_{X^{\prime}} \\
& \equiv g^{*} K_{X}+\left.(n-\mu) E\right|_{X^{\prime}} .
\end{aligned}
$$


Note that a good semiresolution of $X^{\prime}$ also produces a good semiresolution of $X$. Furthermore, semiresolving $X^{\prime}$ will not increase the coefficient of $E$. Since $n-\mu<$ -1 , we conclude that $X$ is not semi log canonical.

Given a morphism $f: Y \rightarrow X$, we denote by $S_{i}(f)$ the locus where the induced morphism on tangent spaces $d f$ drops rank by $i$, i.e.,

$$
S_{i}(f)=\left\{y \in Y \mid \operatorname{rank} d f_{y} \leq \operatorname{dim} Y-i\right\} .
$$

Proposition 4.6. Let $f: Y \rightarrow X \subset \mathbb{P}^{n+1}$ be a finite morphism, and suppose $y \in S_{i}(f)$. Then the point $f(y) \in X$ has multiplicity at least $2^{i}$.

Proof. Since $y \in S_{i}(f)$, the map $d f_{y}: T_{y} Y \rightarrow T_{f(y)} X$ has rank at most $n-i$. To compute the multiplicity of $f(y)$ on $X$, we compute the intersection multiplicity of a general line $L$ with $X$ at $f(y)$. $L$ is determined by $n$ linear forms, say $l_{1}, \ldots, l_{n}$. We can compute the intersection multiplicity by pulling back the $l_{i}$ to $Y$, where they generate $n$ hypersurfaces. Since $d f$ drops rank by $i$ at $y$, no more than $n-i$ of the equations defining these hypersurfaces have independent linear terms at $y$. Without loss of generality, we may assume that the remaining $i$ equations have at least degree 2 at $y$; thus $\left(f^{*} L\right) . Y$ has multiplicity at least $2^{i}$ at $y$. Therefore $f(x)$ also has multiplicity at least $2^{i}$.

Corollary 4.7. Let $X \subset \mathbb{P}^{31}$ be a generic projection hypersurface obtained via $\pi: Y \rightarrow \mathbb{P}^{31}$, where $\Omega_{Y}^{1}$ is nef. Then $X$ is not semi log canonical.

Proof. The hypothesis that $\Omega_{Y}^{1}$ is nef, together with Laz04a, Cor. 7.2.18], implies that $S_{5}(\pi) \neq \emptyset$. Proposition 4.6 implies that for any $y \in S_{5}(\pi)$, the image $f(y)$ has multiplicity at least $2^{5}=32$. The result then follows by Theorem 4.5 ,

Example 4.8. If $Y$ is a smooth projective scheme over $\mathbb{C}$ which is uniformized by $\mathfrak{B}^{n} \subset \mathbb{C}^{n}$, then $\Omega_{Y}^{1}$ is ample, and thus nef (cf. [Laz04b, 6.3.36], [ZL86]).

\section{REFERENCES}

[DB81] P. Du BoIs: Complexe de de Rham filtré d'une variété singulière, Bull. Soc. Math. France 109 (1981), no. 1, 41-81. MR613848 (82j:14006)

[Fed83] R. FEDder: F-purity and rational singularity, Trans. Amer. Math. Soc. 278 (1983), no. 2, 461-480. MR701505 (84h:13031)

[GT80] S. Greco and C. Traverso: On seminormal schemes, Compositio Math. 40 (1980), no. 3, 325-365. MR571055 (81j:14030)

[Har77] R. HaRtshorne: Algebraic geometry, Springer-Verlag, New York, 1977. MR0463157 $(57: 3116)$

[Kol90] J. Kollár: Projectivity of complete moduli, J. Differential Geom. 32 (1990), no. 1, 235-268. MR 1064874 (92e:14008)

[Kol92] J. Kollár ET AL.: Flips and abundance for algebraic threefolds, Société Mathématique de France, Paris, 1992. MR.94f:14013

[Kov99] S. Kovács: Rational, log canonical, Du Bois singularities: on the conjectures of Kollár and Steenbrink, Compositio Math. 118 (1999), no. 2, 123-133. MR:1713307 (2001g:14022)

[Laz04a] R. LAZARSfeld: Positivity in algebraic geometry. I, Ergebnisse der Mathematik und ihrer Grenzgebiete. 3. Folge. A Series of Modern Surveys in Mathematics [Results in Mathematics and Related Areas. 3rd Series. A Series of Modern Surveys in Mathematics], vol. 48, Springer-Verlag, Berlin, 2004, Classical setting: Line bundles and linear series. MR2095471 (2005k:14001a) 
[Laz04b] R. Lazarsfeld: Positivity in algebraic geometry. II, Ergebnisse der Mathematik und ihrer Grenzgebiete. 3. Folge. A Series of Modern Surveys in Mathematics [Results in Mathematics and Related Areas. 3rd Series. A Series of Modern Surveys in Mathematics], vol. 49, Springer-Verlag, Berlin, 2004, Positivity for vector bundles, and multiplier ideals. MR2095472 (2005k:14001b)

[Rob71] J. RoBerTs: Generic projections of algebraic varieties, Amer. J. Math. 93 (1971), 191214. MR0277530 (43:3263)

[Rob75] J. RoberTs: Singularity subschemes and generic projections, Trans. Amer. Math. Soc. 212 (1975), 229-268. MR0422274 (54:10265)

[Sch06] K. SchwedE: On f-injective and Du Bois singularities, Ph.D. thesis, University of Washington, 2006.

[Ste81] J. H. M. Steenbrink: Cohomologically insignificant degenerations, Compositio Math. 42 (1980/81), no. 3, 315-320. MR607373 (84g:14011)

[ZL86] M. G. ZAĬDENBERG AND V. Y. LIN: Finiteness theorems for holomorphic mappings, Current problems in mathematics. Fundamental directions, Vol. 9 (Russian), Itogi Nauki i Tekhniki, Akad. Nauk SSSR Vsesoyuz. Inst. Nauchn. i Tekhn. Inform., Moscow, 1986, pp. 127-193, 272. MR860611

Department of Mathematics, University of Washington, Seattle, Washington 98195

Current address: Department of Mathematics, Seattle University, Seattle, Washington 98122 